\title{
CONTROLLED NANOPARTICLE GROWTH BY VAPOUR CONDENSATION
}

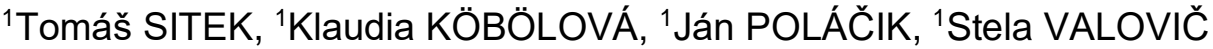 \\ ${ }^{1}$ Faculty of Mechanical Engineering, Brno University of Technology, Brno, Czech Republic, EU, \\ Tomas.Sitek@vutbr.cz, Klaudia.Kobolova@vutbr.cz, Jan.Polacik@vutbr.cz, Stela.Valovic@vutbr.cz
}

https://doi.org/10.37904/nanocon.2021.4369

\begin{abstract}
Domestic boilers are generally characterized by higher emissions of airborne dust. A commonly used secondary method of reducing emissions in the energy sector is a cyclone. However, its wider expansion in households is limited by, among other things, the low efficiency of particle capture below 1 micrometre in diameter, and it is these sizes that dominate in the flue gas of domestic heating devices. By sharply lowering the temperature of the flue gas below the dew point of the vapour, it condenses on all available surfaces. This effect could increase the diameter of the particles, which could be separated with higher efficiency. A change in the numerical distribution of the fine particles with a temperature and thus the supersaturation of the flue gas was sought. The flue gas passed through an impinger filled with water and isopropyl alcohol at three different temperature regimes. The impinger also served to capture the condensate, which was then subjected to morphology analysis using an electron microscope and determination of particle distribution in the condensate.
\end{abstract}

Keywords: biomass, particulate matter, combustion, SEM, SMPS

\section{INTRODUCTION}

In the last few decades, in connection with the environmental impact of combustion processes, attention was primarily paid to gas emissions, but it is currently shifting towards fine particle emissions. Fine particles are a small liquid and solid fraction up to $2.5 \mu \mathrm{m}$ in diameter. In the last few years, the researchers were devoted to submicron and ultrafine particles (up to $0.1 \mu \mathrm{m}$ ), which affect human health significantly. They are so small that the human respiratory tract does not intercept them and they penetrate directly into the alveoli [1,2]. Due to an active surface, ultrafine particles can be much more dangerous than the same mass of larger particles $[3,4]$. Many researchers mention the necessity of changing the current way of emission assessment [5]. The crucial aspects in relation to the impact on human health are the active surface of the particles [6], and their ability to bind toxic substances [7] and penetrate into the lower respiratory tract.

The technologies for fine particle reduction are different. By using a suitable combustion chamber design and selecting the operating parameters we ensure that all the volatile combustible matter remains at a high temperature for a sufficient time so that it gets combusted. However, we know from practice that in real combustion conditions, a certain amount of non-combusted substances get into the atmosphere. Therefore, we are introducing secondary separation of these particles using suitable methods. In the biomass combusting boilers in power or heating plants, secondary separation of dust particles is a matter of course. On the other hand, small biomass boilers in households do not usually use separation technology and their contribution to the total air pollution is considerable [8].

Thus, it is appropriate to look for ways to minimize the number of particles emitted into the air by small combustion devices as well. However, $\mathrm{PM}_{1}$ particles (size up to $1 \mu \mathrm{m}$ ) are difficult to separate by the available methods. HEPA filters can separate particles of this size, but the operation of these devices is associated with high-pressure loss, high cost and frequent maintenance requirements due to intensive clogging [9]. From the 
group of dry separators, an electrostatic precipitator [10] is most often used to capture fine particles. For small combustion plants, however, a major technical complication is to supply the electrostatic precipitator with electricity or provide water for wet separators [11]. A bubble column filled with water is used for wet separation in experimental devices of laboratory scale, as in this study. In a saturated vapour environment, particles may serve as condensation nuclei [12]. In such an environment, these particles grow, and their separation becomes more efficient.

\section{EXPERIMENTAL METHODS}

An automatic wood pellet boiler EcoScroll ALFA, specially adapted for experimental purposes, was used as the experimental combustion device. The nominal thermal output of the boiler is $25 \mathrm{~kW}$. As a fuel, spruce wood pellets ENplus A1 were burned. The moisture content of used fuel was around $6 \%$, ash content was $0.3 \%$ of raw biomass. The proximate analysis was conducted by the authors according to the European standards EN ISO 18134-3 and 18122. During the tests, the boiler worked at its nominal output with continuous fuel feed. A sample of flue gas from the boiler at approximately $140{ }^{\circ} \mathrm{C}$ was taken using a sampling probe located in the flue gas stack, at a height of $1 \mathrm{~m}$ above the outlet of the combustion device (see Figure 1). This point was selected in compliance with the standard requirements (EN 13240) for measuring emissions in small combustion devices. Oxygen content in the flue gas fluctuated from 10.0 to $11.5 \%$, and thus the results were converted to reference oxygen content (10\%).

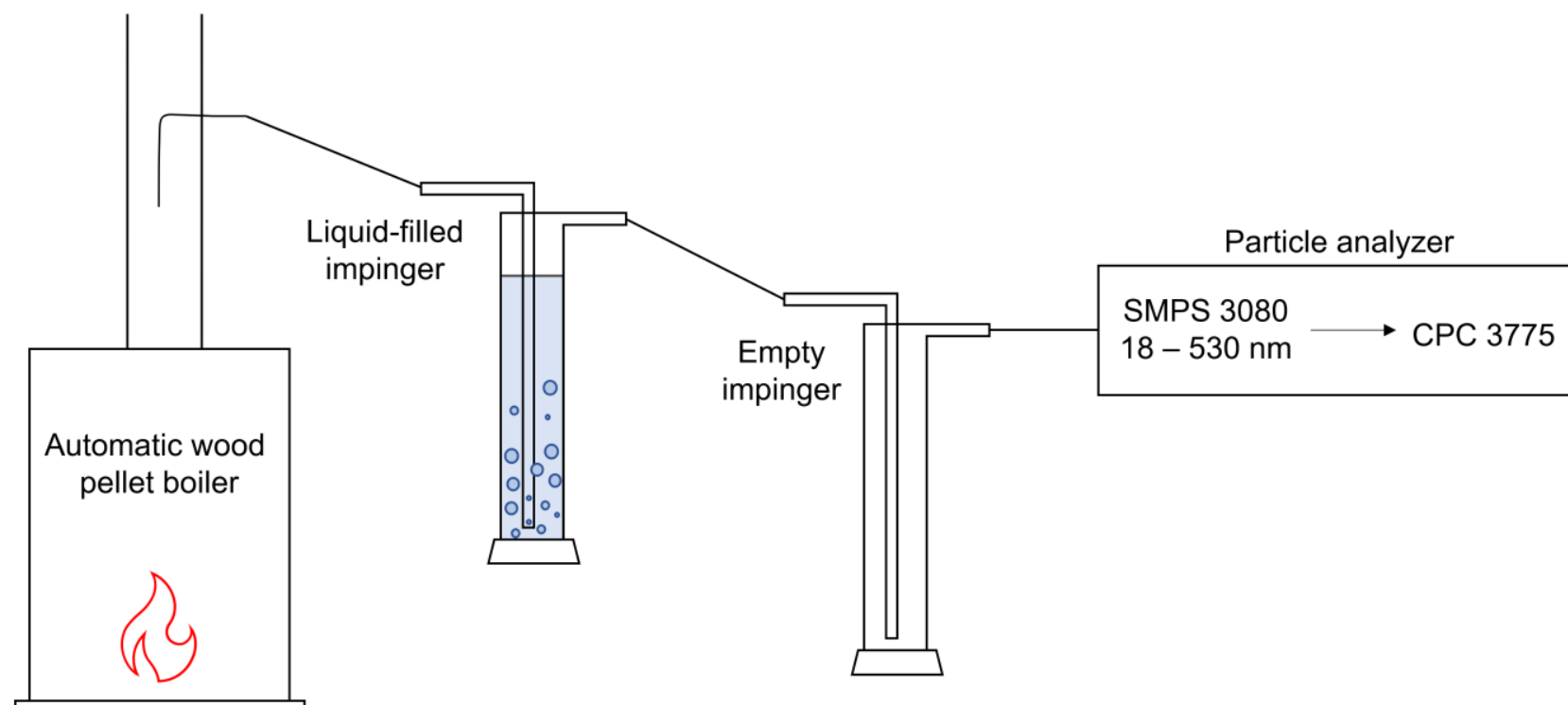

Figure 1 Scheme of experimental setup

The flue gas with dispersed fine particles was carried from the stack through the short tube directly to the first impinger. This $250 \mathrm{ml}$ impinger was filled with $200 \mathrm{ml}$ of water or isopropyl alcohol (so the gas had to pass through approximately $12 \mathrm{~cm}$ of liquid) and submerged into the water bath. To cool flue gas down, brine solution $\left(-20^{\circ} \mathrm{C}\right.$ at the beginning, but gradually warming up to $\left.-8{ }^{\circ} \mathrm{C}\right)$ was used. To warm flue gas up, hot water was used, which progressively cooled down from $85{ }^{\circ} \mathrm{C}$ to $55^{\circ} \mathrm{C}$. Due to the very low sampling volume ( 0.3 litres per minute), it was arduous to directly measure the temperature of the flue gas past the impinger, as it cooled rapidly at the outlet. Nevertheless, we assume the minimal temperature difference between the water bath and flue gas. Subsequently, the gas was carried downwards to second (again $250 \mathrm{ml}$ ), empty impinger to collect potential condensate. After that, flue gas was carried directly to the measuring device. Empty impinger without any liquid was used for comparison as well. 
For particle concentration and distribution measurements, Scanning mobility particle sizer (SMPS 3080) and Condensation particle counter (CPC 3775) devices by TSI Inc. were used. Unseparated particles with electrical mobility diameter from 18 to $530 \mathrm{~nm}$ were monitored for 60 minutes in each setting.

The morphology of the particles was observed using a scanning electron microscope (SEM), particularly the LYRA 3 (Tescan) electron microscope. For the SEM measurement, the sample was covered with a conductive layer of gold with a thickness of $20 \mathrm{~nm}$ using a planetary rotation of the samples. A detector of secondary electrons (SE) was used for the measurements. The elemental composition of the particles in the condensate was analysed by the ICP-OES spectrometer ARCOS by Spectro.

\section{RESULTS AND DISCUSSION}

Initially, the empty impinger setting at room temperature was tested to obtain the boiler's original particulate emissions. Due to the randomness of combustion processes, 60 one-minute measurements were averaged. Results of average particle number concentration are shown in Figure 2, the uncertainty is expressed as a single standard deviation.

At room temperature, both water and isopropanol cause a decrease of number concentration by $22 \%$ compared with empty impinger. Different temperature leads to another decrease with ambiguous trends. In the case of water, cooling the gas down is more effective and results in supersaturation and particles with condensed water vapour are collected in the brine solution. On the other hand, hot water creates gas with higher absolute but lower relative humidity. In this case, supersaturation is achieved as soon as flue gas cools down, which is somewhere between the two impingers.

Cold isopropanol does not seem to do much of a difference while warming it is the most effective method regarding particle separation. Hot isopropanol separated $65 \%$ of the original particle number concentration. The temperature of the hot water bath was very close to the boiling point of isopropanol, which caused a considerable amount of isopropanol to evaporate and condensate in the tube leading downwards to the second impinger. The wet inner surface of the tube and condensation of liquid after cooling are probably the main factors.

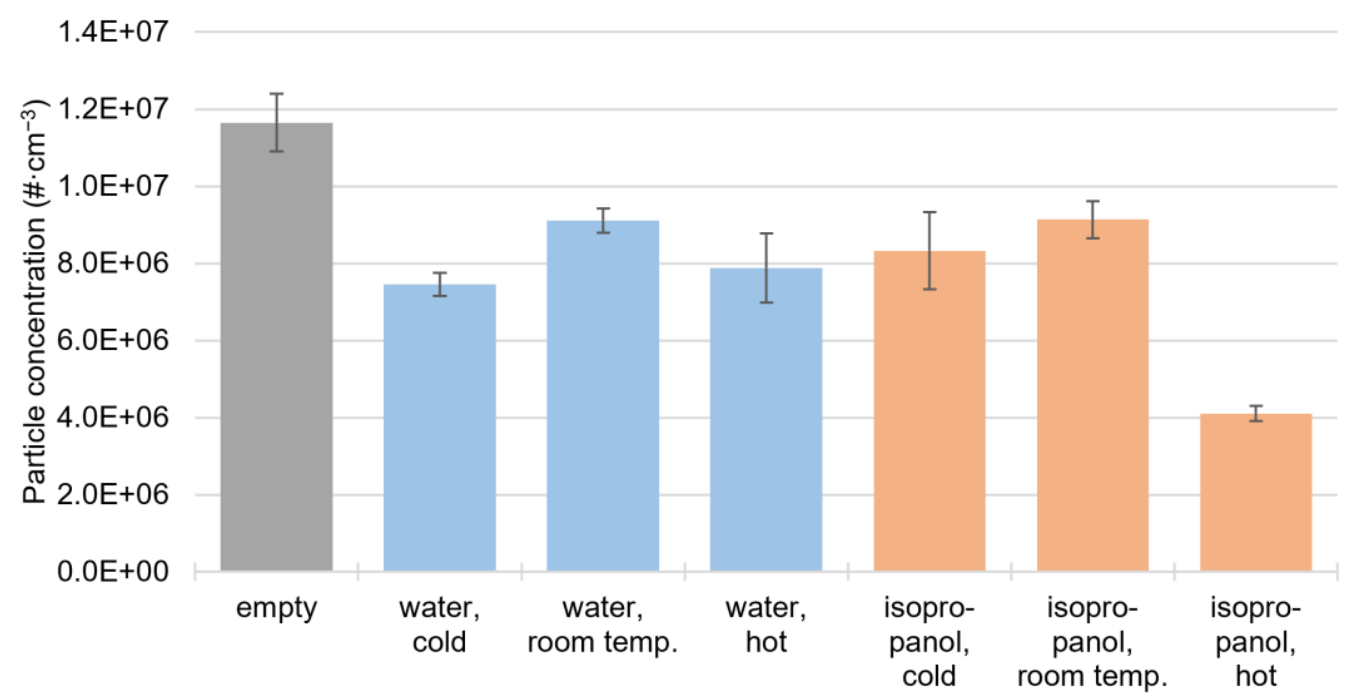

Figure 2 Average particle number concentrations

Particle diameters slightly increased after passing through the water compared to the empty impinger (Figure 3). In the cold bath, the particles grew more than in the hot bath or at room temperature conditions; the latter two setups were rather similar. 
Isopropanol and water curves at room temperature are almost indistinguishable. Cold bath again caused the biggest increase in size, while the hot bath is the only setup, that affected the distribution noticeably and caused diameters to decrease. When the effect of lower particle concentration is disregarded, it is clearly visible, that much more of the larger particles were separated. There is even a decrease in the number of smallest particles, which were unaffected by other settings. Temperature does not appear to have a significant impact on particle sizes and the size changes are more likely due to the more probable separation of larger particles.

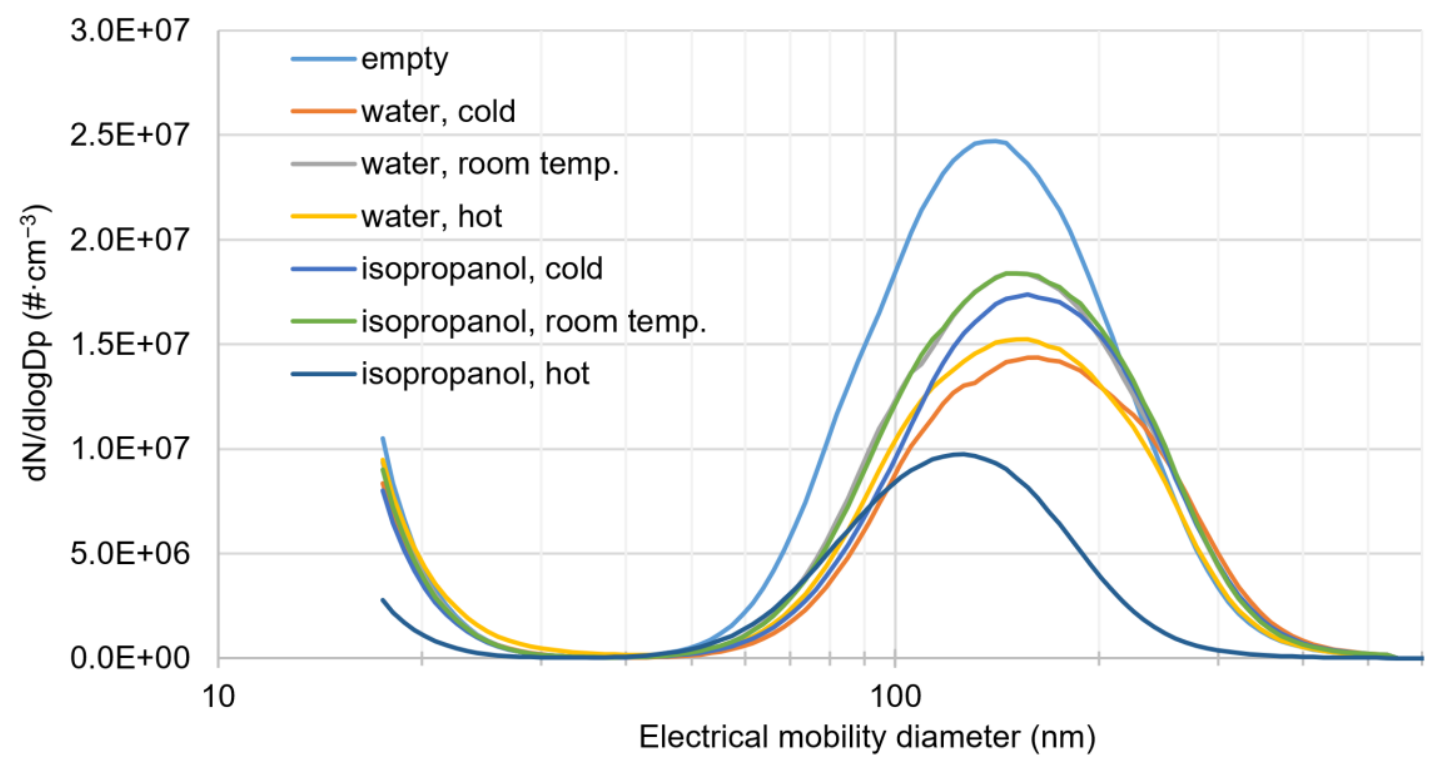

Figure 3 Average particle distributions
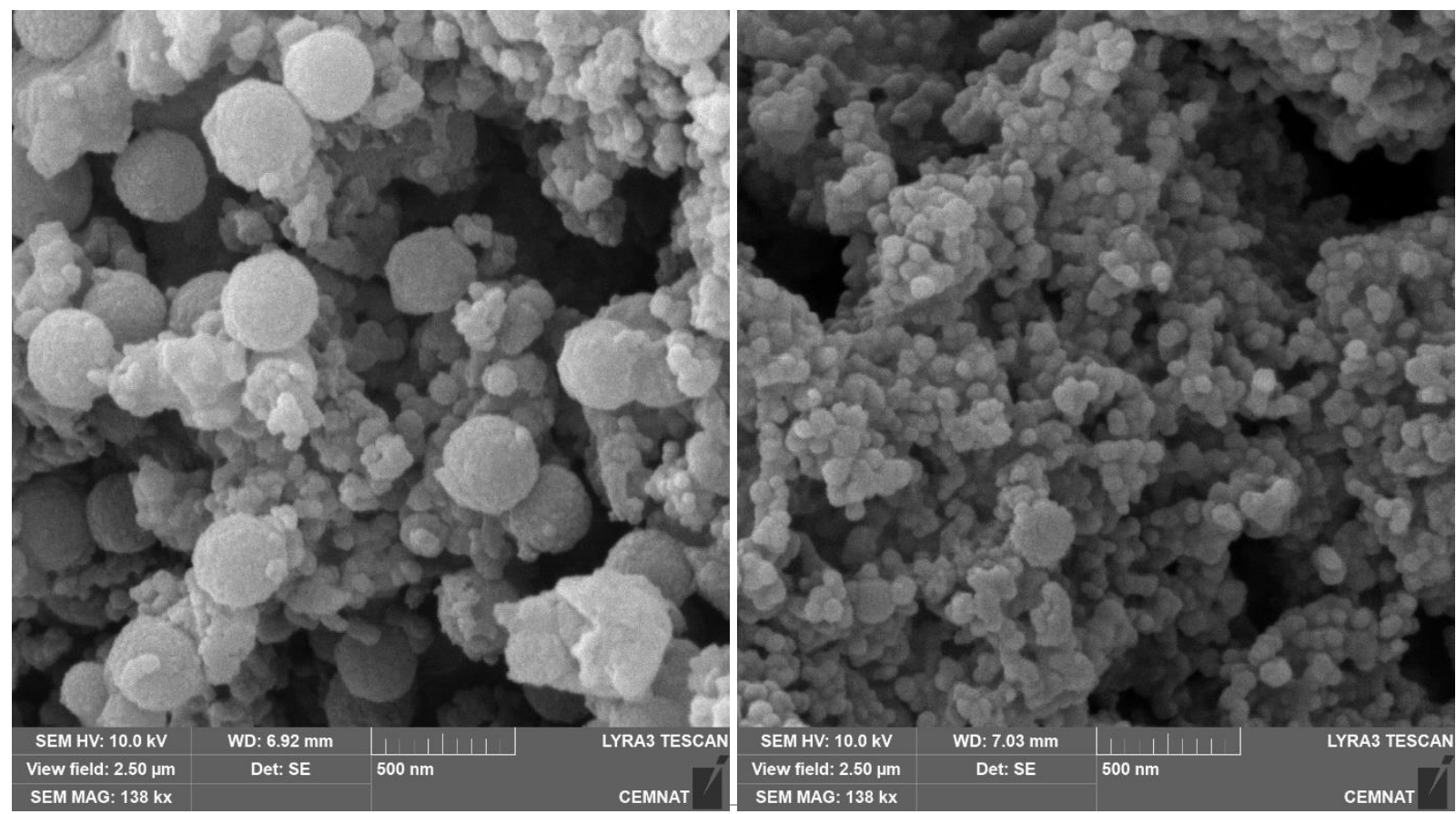

Figure 4 Morphology of particles in condensate with a magnification of $138000 \times$, measuring scale is in the pictures

A sample of the condensate with captured particles from the second impinger was slowly dried and analysed by SEM. There are two types of particles observed in the condensate - spherical to oval nanoparticles and 
regular spherical macroparticles (see Figure 4). The size of the nanoparticles ranges from 45 to $70 \mathrm{~nm}$ and that of the spherical macroparticles ranges from 300 to $350 \mathrm{~nm}$. Both types of particles are agglomerated together into a three-dimensional structure, but nanoparticles are sometimes fused together almost beyond recognition. It is not known, how much was the morphology affected by the thickening of the condensate, i.e., how particles looked before being separated. The number of spherical macroscopic particles in the sample is considerably smaller. The exact composition of spherical particles is unknown, but their shape indicates phase change (solidification) in flight.

Concerning metals content, the most dominant was potassium and calcium, in smaller amounts were detected also manganese, magnesium and sodium. These results are no surprise, as they account for most of the inorganic content in used biomass. Note that the used measuring device is unable to detect carbon and gases (e.g., oxygen, nitrogen, and hydrogen), and carbon and oxygen in particular normally constitute a considerable part of particle mass [13].

\section{CONCLUSION}

The experiments proved that the number of fine particles of 18 to $530 \mathrm{~nm}$ in diameter in the flue gas can be reduced by passing the gas through a liquid-filled impinger with significant efficiency. There is almost no difference when brine solution or isopropanol is used as a scrubbing medium at room temperature. Both heating and cooling these liquids results in additional improvement of particle separation. The highest particle separation efficiency of $65 \%$ was achieved with isopropanol heated to temperatures just below its boiling point. Condensation of isopropanol on particles and intense wetting of surfaces caused even the smallest particles to be captured. In a practical operation, however, usage of a flammable and irritant substance is not optimal and usage of regular water is much more promising. Heating water to its boiling point could have a similar effect.

Regarding morphology of particles collected in the condensate, the typical soot structures around $60 \mathrm{~nm}$ in diameter were found as well as larger spherical particles with diameter up to $350 \mathrm{~nm}$. By analysing metal content, similar elements as in the original biomass were found, such as potassium and calcium.

The original idea of increasing particle diameters by condensing liquid in their surfaces turned out to be more complicated than expected. Condensation did occur, but too little for any practical application. It could be caused by preferred condensation on surfaces of tubes, which are colder than aerosol particles. If this is the case, usage of larger diameter tubes would be beneficial.

\section{ACKNOWLEDGEMENTS}

This paper was supported by TA ČR within the TJ04000415 project of System for Separation of Fine Particles from Flue Gas Stream During Biomass Combustion and Research Centre for Low-Carbon Energy Technologies funded as project No. CZ.02.1.01/0.0/0.0/16_019/0000753 by Czech Republic Operational Programme Research, Development and Education, Priority 1: Strengthening capacity for high-quality research and the collaboration. This research was further funded by projects of Brno University of Technology: FSleS-20-6280.

\section{REFERENCES}

[1] ROM, William N., MARKOWITZ, Steven MD. Environmental and Occupational Medicine. 4. Philadelphia, PA: Lippincott Williams \& Wilkins, 2007. ISBN 9780781762991.

[2] U.S. EPA. Air Quality Criteria for Particulate Matter (Final Report, April 1996). U.S. Environmental Protection Agency, Washington, D.C., EPA 600/P-95/001. Available from:

http://cfpub.epa.gov/ncea/risk/recordisplay.cfm?deid=2832. 
[3] WANG, Kun, WANG, Weiye, LI, Lili et al. Seasonal concentration distribution of PM1.0 and PM2.5 and a risk assessment of bound trace metals in Harbin, China: Effect of the species distribution of heavy metals and heat supply. Scientific Reports. [online]. 2020, vol. 10, no. 1. ISSN 2045-2322. Available from:

https://doi.org/10.1038/s41598-020-65187-7.

[4] MAINKA, Anna and ZAJUSZ-ZUBEK, Elwira. PM1 in Ambient and Indoor Air-Urban and Rural Areas in the Upper Silesian Region, Poland. Atmosphere. [online]. 2019, vol. 10, no. 11. ISSN 2073-4433. Available from: https://doi.org/doi.org/10.3390/atmos10110662.

[5] MERKISZ, Jerzy, PIELECHA, Jacek. The Relationship between Particle Mass and Particle Number. In: Nanoparticle Emissions From Combustion Engines. [online]. 2015, pp. 93-108. ISBN 978-3-319-15927-0. Available from: https://doi.org/10.1007/978-3-319-15928-7 8.

[6] PARK, Minhan, JOO, Hung Soo, LEE, Kwangyul, et al. Differential toxicities of fine particulate matters from various sources. Scientific Reports. [online]. 2018, vol. 8, no. 1. ISSN 2045-2322. Available from: https://doi.org/10.1038/s41598-018-35398-0.

[7] SCHMID, Otmar, STOEGER Tobias. Surface area is the biologically most effective dose metric for acute nanoparticle toxicity in the lung. Journal of Aerosol Science. [online]. 2016, vol. 99, pp. 133-143. ISSN 00218502. Available from: https://doi.org/10.1016/j.jaerosci.2015.12.006.

[8] WEIMER, S., ALFARRA, M. R., SCHREIBER, D., MOHR, M., PRÉVÔT, A. S. H. and BALTENSPERGER, U. Organic aerosol mass spectral signatures from wood-burning emissions: Influence of burning conditions and wood type. Journal of Geophysical Research. [online]. 2008, vol. 113, no. D10. ISSN 0148-0227. Available from: https://doi.org/10.1029/2007JD009309.

[9] HWANG, Sungho, ROH, Jaehoon and PARK, Wha Me. Comparison of the relative performance efficiencies of melt-blown and glass fiber filter media for managing fine particles. Aerosol Science and Technology. [online]. 2018, vol. 52, no. 4, pp. 451-458. ISSN 0278-6826. Available from: https://doi.org/10.1080/02786826.2017.1423274.

[10] YUAN, Fangyang, YU, Jianfeng and ZHENG, Xiangyang. Experimental study on fine particle separation in a wet electrostatic classifier. Chemical Engineering and Processing - Process Intensification. [online]. 2020, vol. 156. ISSN 02552701. Available from: https://doi.org/10.1016/j.cep.2020.108108.

[11] BIANCHINI, Augusto, PELLEGRINI, Marco, ROSSI, Jessica and SACCANI, Cesare. Theoretical model and preliminary design of an innovative wet scrubber for the separation of fine particulate matter produced by biomass combustion in small size boilers. Biomass and Bioenergy. [online]. 2018, vol. 116, pp. 60-71. ISSN 09619534 Available from: https://doi.org/10.1016/i.biombioe.2018.05.011.

[12] CUI, Lin, SONG, Xiangda, LI, Yuzhong, WANG, Yang, FENG, Yupeng, YAN, Lifan a DONG, Yong. Synergistic capture of fine particles in wet flue gas through cooling and condensation. Applied Energy. [online]. 2018, vol. 225, pp. 656-667. ISSN 03062619. Available from: https://doi.org/10.1016/j.apenergy.2018.04.084.

[13] JONES, Jenny M, LEA-LANGTON, Amanda R., MA, Lin, POURKASHANIAN Mohamed a WILLIAMS, Alan. Pollutants Generated by the Combustion of Solid Biomass Fuels. [online]. 2014. Available from: https://doi.org/10.1007/978-1-4471-6437-1. 